This item was submitted to Loughborough's Research Repository by the author.

Items in Figshare are protected by copyright, with all rights reserved, unless otherwise indicated.

\title{
Benefits of stochastic optimisation with grid price prediction for electric vehicle charging
}

PLEASE CITE THE PUBLISHED VERSION

https://doi.org/10.4271/2017-01-1701

\section{PUBLISHER}

(C) SAE International

\section{VERSION}

AM (Accepted Manuscript)

\section{PUBLISHER STATEMENT}

This work is made available according to the conditions of the Creative Commons Attribution-NonCommercialNoDerivatives 4.0 International (CC BY-NC-ND 4.0) licence. Full details of this licence are available at: https://creativecommons.org/licenses/by-nc-nd/4.0/

\section{LICENCE}

CC BY-NC-ND 4.0

\section{REPOSITORY RECORD}

Mody, Sagar M., and Thomas Steffen. 2018. "Benefits of Stochastic Optimisation with Grid Price Prediction for Electric Vehicle Charging”. figshare. https://hdl.handle.net/2134/34007. 


\section{Benefits of Stochastic Optimisation with Grid Price Prediction for Electric Vehicle Charging \\ S. Mody, T. Steffen}

Loughborough University

\begin{abstract}
The goal of grid friendly charging is to avoid putting additional load on the electricity grid when it is heavily loaded already, and to reduce the cost of charging to the consumer. In a smart metering system, Day Ahead tariff (DA) prices are announced in advance for the next day. This information can be used for a simple optimization control, to select to charge at cheapest times. However, the balance of supply and demand is not fully known in advance and the Real-Time Prices (RTP) are therefore likely to be different at times. There is always a risk of a sudden price change, hence adding a stochastic element to the optimization in turn requiring dynamic control to achieve optimal time selection. A stochastic dynamic program (SDP) controller which takes this problem into account has been made and proven by simulation in a previous paper.
\end{abstract}

Since there are differences between the DA and the RTP tariff, this paper proposes a (1) predictor to create an unbiased estimate of the RTP tariff based on available data. It uses a regression on historical data to find the best prediction of the expected price. Finally, a (2) case study based on data from the Illinois Electricity Grid prices is presented to validate the SDP controller over several years of data. The stochastic optimization uses the RTP prices effectively, getting very close to the globally optimal charging price. However, the predictor achieves only a slight reduction in prediction uncertainty with this data sate, and it has a negligible effect on cost. This means that DA prices can be used as a fair prediction of RTP charging cost here. The SDPM successfully reacts in the case study and leads to savings on charging costs over the years presented.

\section{Introduction}

The goal of reducing carbon and greenhouse emissions, has created a revolution in the automotive industry, leading to the popularity of Electric Vehicles (EVs). The initial adoption of EVs, whether Hybrid, Plug-In or pure Battery powered EVs has been mild, with Toyota, the major producer of the pioneering Hybrid EV Prius selling 2 million units by 2009. Many studies in the last decade have claimed that market forces alone have not been able to make the EV a first choice for many consumers; government policy support, research to make EVs less expensive and economy improvement will improve their market significantly [1][2]. This has been generally true, as there are many more adopting the EV with the introduction of better battery technology and more mainstream vehicle manufacturers, like Nissan and BMW, deciding to sell EVs. The other important concern that vehicle buyers have is range anxiety. With an 'everyday' EV that is affordable like the Nissan LEAF, only being able to promise a theoretical 84 miles [3], many buyers don't see the value of a more expensive car of the same size. However, surveys have indicated that

Page 1 of 12
$47-55 \%$ of single vehicle usage in a single day is less than 20 miles, with $82-88 \%$ of vehicles travelling less than 60 miles [4]. Kang and Recker's 2009 [4] study concludes that it is possible to convert between $80 \%$ to $90 \%$ of daily mileage to electric when using PHEV with a 60 mile range in California; under the condition that both home and public place charging stations are in use. EVs like Tesla, are also becoming more popular, making EVs more likeable to the driving enthusiast and boasting a better battery technology and significantly more minimum mileage of 199 miles[5].

As EVs become more mainstream, it is logical to conclude, that more their numbers, more will be the impact on the Electricity Grid when they are charging. The electricity grid and systems must be prepared for the influx of the extra recharging load and also look at it as an opportunity for vehicle to grid (V2G) solutions. The batteries of these vehicles are large and require long times, high power and high currents for charging. For most domestic users, charging will take place overnight at their homes. Moreover, the tendency to plug the vehicle in as soon as they reach home is high. Numerous studies like [6]-[10] highlight the impact of EVs on electricity distribution in the future, in elaborate detail. Whilst the recharging load for a small number of EVs is likely to be buried in the baseline load fluctuation, a large fleet of EVs charging at the same time could have an overwhelming effect on the grid during peak hours [11]. Therefore, EVs are already becoming a stakeholder in the 'Electricity System'. Subsequently, the electricity market, generation methods and infrastructure will need improving, adapting and expanding to support the influx of the new stakeholder.

Following on from the discussion, we can conclude that the electricity grid and EV will be interdependent in the future. Firstly, it will be important to manage the electricity loads and peak demands due to user profile of EV charging. Some load may have to be shifted from peak hours either by persuading or enforcing the consumer to charge earlier or later. Secondly, charging the vehicle without control might also be a disadvantage for the consumer due to the possibility of a future with RTP. There is a good argument for vehicle to grid solutions (V2G) to help reduce the impact on the electricity grid in a future where there are high numbers of EVs charging at the same time. However, the more immediate solution to consider in the same scenario, is a smart automated charging control, whereby the electricity cost of charging is minimized to the vehicle user, by delaying the charging to the hours when there is already less stress on the electricity grid.

In a previous paper [12], a stochastic optimization algorithm based strategy was discussed and proven by simulation to successfully minimize the total cost of charging over a given period. The optimal controller based on a stochastic dynamic program (SDP), which predicts future price changes from available data. The controller takes 
into account price variability via a simple grid model that allows of unexpected price rises and a gradual return to a normal grid price. The DP algorithm has two variables, the state of charge (SoC) and the current electricity cost. It traces the expected total cost based on the stochastic model and makes a decision 'to charge or not' to minimize the expected (average) total cost.

This paper uses real data from the Illinois price grid, provided by ComEd, to perform a case study and prove the advantage of using the aforementioned Stochastic Dynamic Programming Strategy (SDPM). Secondly, this paper also looks at the electricity spot-price or dayahead market prices using the same data, to perform a linear regression analysis, to make a basic 'predictor' to try and improve the day-ahead price prediction (DAP) which has already been provided by the electricity company.

This paper is split into the following sections: An overview of the research and previous publications is summarized. Secondly, some literature is presented on the day ahead electricity price market and grid price prediction strategies. Following this is the linear regression study of the data from the Illinois grid. Finally the proof and case study of the SDP controller are presented with a brief summary of the controller.

\section{Overview and Contribution of this research}

This paper is a part of a $\mathrm{PhD}$ which aims to achieve an optimal control strategy for charging of an EV, with the goal to reduce the overall cost to the consumer, hence offsetting the charging from the peak hours and in turn automate this procedure. It has been split into three parts, the first two of which have already been published.

1. The first explains the problem and a deterministic (nonoptimal) solution to it [13]. A case study for the deterministic controller is also explained in the paper. It concludes that, if prices are known in advance (day-ahead pricing), the optimization only requires picking the cheapest time slots for charging the battery

2. The second part, progresses the above study and proposes an optimal controller based on a stochastic dynamic program (SDP), which predicts future price changes from available data. Its presents the proof of this optimal controller by simulation [12]. It also presents a literature study.

3. The third part is presented in this paper. A case study proof of the controller explained in paper [12] and this paper also looks at the day-ahead market prices using the same data, to perform a linear regression analysis.

The SDPM optimal controller, presents a unique approach in which we don't aim to establish a hierarchical system of control but we look at the problem from the perspective of the vehicle owner. We aim to keep the computational complexity low with a future view of fitting the strategy into an embedded device. Paper [12] explains this in detail with a simulation. It also covers a more in depth literature study of similar approaches, driver charging behavior and explanations of why the charging profile used to prove the controller has been chosen. In this study, The stochastic nature of the problem is realized and a better and optimal solution is presented compared to the sub-optimal controller presented in part 1[13]. The prediction ability of the controller has been tested using simulation of a 14 hour period of a set of arbitrary prices. The controller behaves as expected and not only predicts possible price jumps but also reacts to by being able to provide required SoC thus not leaving the user stranded.

\section{This paper carries on from the previous study and presents:}

1. A case study with real price data from ComEd, Illinois and proves the validity of the SDPM, highlighting the potential financial benefit of this approach to the vehicle owner.

2. A linear regression analysis of the price data, with the aim to quantify the validity of the provided Day Ahead Price Prediction from ComEd versus the real time price data and to possibly improve upon it.

\section{Day Ahead Electricity Price and Price Prediction}

In industrial sectors, the 'spot-electricity' market price-forecasting techniques are relied upon for bidding purposes and hedging against volatilities. With a good next day forecast, a stakeholder in the 'Electricity System' would be able to make better financial decisions. A power producer could develop strategies to maximize its pay off and the consumers could minimize their utilization costs [14]. As such, there are three types of price forecasting, short-term, mid-term and long-term. The one to consider here is the short-term period, hourly and daily.

Many factors influence the electricity prices. The cost of fuel used to produce electricity, the transmission and distribution systems of the power plants, weather conditions, the load on the grid depending on the location and location specific regulations are just some important considerations. Even basic factors like day, hour, week and month are of importance, when the reaction of the consumers is taken into account. Electricity grids can also suffer transmission congestion which may prevent free power exchange between control nodes. This creates complex non-linearity in the electricity load and prices, making them even more difficult to predict. This volatility can give rises to unexpected electricity price spikes.

There are too many studies and predictor proposals to list; they range in a multitude of approaches. Han et al. [15] propose a composite approach for ultra- short term load forecasting using two well-known methods: recursive least square support vector machine algorithm and Takagi-Sugeno fuzzy control. Hong, Wilson and Xie [16] propose statistical methods to predict long term probabilistic forecasts, also including linear regression with multiple factors. They use hourly information to create a more robust forecast using predictive modelling, scenario analysis and weather data. Singhal et al. [17] use back propagation with neural networks. Their basic idea uses history and other estimated factors in the future to fit and extrapolate, to achieve a price prediction. Many of these studies and the strategies they propose, are already being used by power utilities and claim to have less than 5\% error in their predictions [15]-[17]. In spite of the numerous methods in use and ongoing research studies short term price forecasting isn't a mature science, especially with price sensitive loads being introduce in the system [18].

The electricity market, like any other is dependent on the economics of supply and demand. With the introduction of smart-grids and smart-tariffs, the price of electricity to the regular user will no longer be a static cost per which they are charged. They too can benefit from dynamic information about price and change their usage to suit. In most countries, the industrial sectors are on wholesale electricity

Page 2 of 12 
prices- buying electricity at lower rates during off-peak hours. In case in Portugal, Germany, some parts of continental Europe and a few states in the USA, smart-meter systems allow this benefit to consumers. In such markets, the consumers are encouraged to shift their electricity usage to off-peak hours through high-price updates/alerts either hourly or daily. Advances in 'Smart-Grid' technology can allow this elastic behavior from households, helping them to reduce costs. Electric utility providers in California, Colorado, Florida, Illinois, Indiana, Ohio, Texas, Washington and some other states have already been introducing smart grids to many customers. There is also a strong financial incentive being provided for both smart grid research and introduction via the Energy Independence Act of 2007 and the US Stimulus Package of 2009 [19]. The report [20], claims that $9 \%$ of peak demand could be offset using small-incentive demand response programs, if they were used all over U.S.A.. Moreover, if dynamic pricing programs are introduced to all electric consumers, $20 \%$ of the peak demand could be offset.

Instead of the fixed tariff used by a conventional meter, smart meters can deal with frequently changing tariffs. Typically the cost of electricity charged changes every hour or half an hour, and the electricity company communicates either the current tariff or the expected tariff development for the next day to the customer. This means that customers can move electricity intensive activities into periods where electricity is plentiful, therefore cheap. This reduces the strain on the electricity grid both in terms of distribution and in terms of generation. Once, a significant share of electricity is generated from (generally uncontrollable) renewable sources, load shifting will be an important measure needed to align generation and consumption.

The state of Illinois is a good example where RTP has been available to customers since 2003. The RTP programs have been successful in reducing the participating consumers' electric usage and bills and shifting usage to non-peak times of the day [21]. The two electricity providers which allow the choice of RTP are Amaren and ComEd. Amaren's Power Smart Pricing (PSP) and ComEd's Residential Real Time Pricing (RRTP) programs have reduced their peak demand in the range of $15 \%$ and achieved participant bill savings between 10$15 \%[21]$.

\section{Linear Regression Day Ahead Predictor}

The question of 'when to charge' is strongly dependent on the knowledge of electricity prices. The idea of price prediction charge automation is based on the availability of hourly price information from the electricity providers to the customer. In markets like Illinois, USA, the two major electricity providers provide tariffs under which the customers a provided price information the day before (DAP) or the hour before (RTP). Retrospectively, both these prices are available from the company. The data available from the electricity provider Amaren, Illinois (year 2010) was first being used as the baseline. However, due to changes in their company policy data from other years was no more available to download and data was downloaded instead, from the electricity provider, ComEd, Illinois. All the data used is from $1^{\text {st }}$ of January of the relevant year to the $31^{\text {st }}$ of December of the same year [22].These prices exclude the distribution cost, which is constant and therefore not relevant for comparison purposes.

So, that question, 'When to charge?' comes down to answering this one, 'How much can we rely on the DAP data for the prediction of the real prices of the next day?'

Page 3 of 12
Looking at the pricing information from a statistical point of view reveals a few surprises as shown in Tables 1 (Amaren, 2010) and 2 (ComEd, 2010). First of all, both the day-ahead price and even more so, the real-time price become negative at times. The standard deviation of the real-time price is much higher than the day-ahead price. The difference between the day-ahead price and the real-time price can be seen as prediction error and interestingly its standard deviation is only slightly lower than standard deviation of the realtime price. Certainly, the latter are much more volatile - and therefore more interesting for load shifting. The correlation coefficient between day-ahead prices and real time prices of the Amaren data is $\mathbf{0 . 4 3}$, which indicates that day-ahead prices have only moderate value as a prediction of the real-time prices. However, the correlation coefficient of the ComEd data is $\mathbf{0 . 7 1}$, indicating that their day-ahead prices have a higher value as a prediction for the real prices.

Table 1: Statistical Properties of Amaren data (2010)

\begin{tabular}{|l|l|l|l|l|}
\hline & Mean & SD & Min & Max \\
\hline Day-Ahead & 2.73 & 1.04 & -0.19 & 19.81 \\
\hline Real Time & 2.63 & 2.20 & -8.85 & 107.58 \\
\hline Difference & -0.10 & 1.99 & -99.19 & 13.19 \\
\hline
\end{tabular}

Table 2: Statistical Properties of ComEd Data (2010)

\begin{tabular}{|l|l|l|l|l|}
\hline & Mean & SD & Min & Max \\
\hline Day-Ahead & 3.61 & 1.60 & -0.44 & 12.38 \\
\hline Real Time & 3.61 & 2.19 & -12.58 & 33.30 \\
\hline Difference & 0.13 & 1.54 & -23.85 & 13.31 \\
\hline
\end{tabular}

As discussed, there are many in-depth studies on 'predictors for hourly electricity prices' and many of these predictors proposed, are already being used by electricity providers themselves. Use of a sophisticated algorithm to generate the DAP is crucial, with multiple underlying factors involved including history and weather predictions. Therefore, the exercise to create this linear regression to make a predictor is to check validity of the DAP provided, and to see if a marginally better prediction can aid the SDPM controller in making a control decision of 'when to charge?' Secondly, there is an underlying question to consider: Are the DAP provided, already tailored in some way by the electricity providers to encourage the users to shift electricity and consequently to affect RTP?

Evidently, the relationship between the DAP and RTP is linear as proven by the correlation but the proper linear regression technique also confirms this. A study has been performed using the standard methodology of the multiple regression technique, to achieve possible predictors on this basis. RTP is treated as the dependent or response variable, which is dependent on the available factors, (available from the data provided by the electricity company) DAP, day of the week, hour of the day and month of the year. These are 
treated as the explanatory or predictor variables. Data for the year 2010 has been used as training data, and the years 2011-2014 have been used for verification.

The linear model underlying the regression analysis is:

$$
y_{i}=\beta_{0}+\beta_{1} X_{i 1}+\beta_{2} X_{i 2}+\cdots+\beta_{p} X_{i p}+\varepsilon_{i}, \quad i=1, \ldots, n,
$$

Equation 1

- $y_{i}$ is the $\mathrm{i}^{\text {th }}$ response

- $\quad \beta_{k}$ is the $\mathrm{k}^{\text {th }}$ coefficient, where $\beta_{0}$ is the constant term in the model

- $X_{i j}$ is the $\mathrm{i}^{\text {th }}$ observation on the $\mathrm{j}^{\text {th }}$ predictor variable, $j=1$, ..., $p$

- $\varepsilon_{i}$ is the $\mathrm{i}^{\text {th }}$ noise term indicating the random error

Since DAP is provided as a reliable prediction by the electricity company, it is considered to be the most important factor in the prediction of RTP for the study. Therefore, starting with the simple model, which uses only DAP as the predictor the following model is obtained for the training data:

Referring to equation $1, y$ is RTP and $X_{l}$ is DAP,

$$
R T P=0.0008+0.9757(D A P)
$$

Table 3: Coefficients of the Simple Model (DAP Predictor)

\begin{tabular}{|c|c|c|c|c|}
\hline & Estimate & Std Error & tStat & pValue \\
\hline Intercept & 0.00086454 & 0.0004056 & 2.1315 & 0.33075 \\
\hline DAP $\left(\boldsymbol{X}_{\boldsymbol{I}}\right)$ & 0.9757 & 0.010272 & 94.988 & 0 \\
\hline
\end{tabular}

Number of observations: 8760, Error degrees of freedom: 8758 RMS error: 0.0154

The results show that DAP is very closely related to RTP in this set of data. As such, DAP is a good prediction of the RTP except in cases 0.15 cents of a difference or in case of very large price spikes. Some outliers and deviations are expected, since the nature of the electricity price is non-Gaussian in nature with a rather long tail, at both very high and very low probability bounds. This can make relying entirely on the linear regression data analysis, quite difficult.

For the most part, the fit is linear with good confidence bounds which conform to the linearity. Whilst, all the different coefficient values obtained are helpful in making a decision about the predictor's usefulness, the most important observations for our purpose are the intercept, coefficient of the DAP factor and the root mean squared (RMS) error estimate. Clearly, the RTP prediction is almost closely based on the DAP with a small error. An attempt to make the 'prediction' better, i.e. attempt to make the linear fit better, is made by trying to use a higher order DAP factor model. Using the second order model:

$$
\boldsymbol{R T P}=-0.0025774+1.1602(\boldsymbol{D A P})-2.065\left(D A P^{2}\right)
$$

RMS: 0.0153

The reduction in the RMS error is negligible, although as expected, it is lower. The coefficients of the model now show a negative proportionality with the higher order DAP factor. Aside from third

Page 4 of 12 order DAP factor model, a full factor model has also been explored. The full model includes all the available factors (hour, weekday, month, DAP), including the interdependencies between them making it a model with 11 terms and 4 predictors. Figure 1 shows the RMS error of the different models compared to that of the original training data.

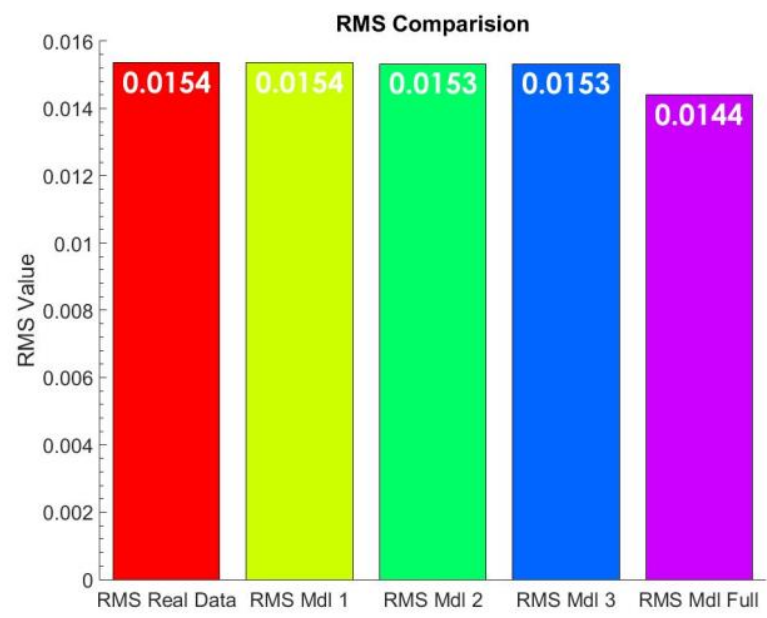

Figure 1: RMS errors of models for training data (2010)

The results show that there is an improvement in the model when multiple factors are added, at least based on the RMS error which shows a reduction compared to the models which only use DAP as a predictor. Although, it would not be correct to conclude that they are fully important because their interactions can be redundant at many values and their coefficients are highly negative in some cases. On the other hand it would be wrong to assume they are unimportant because the effect of hours, days and months is significant to weather and other user/consumer related interactions which do affect electricity prices.
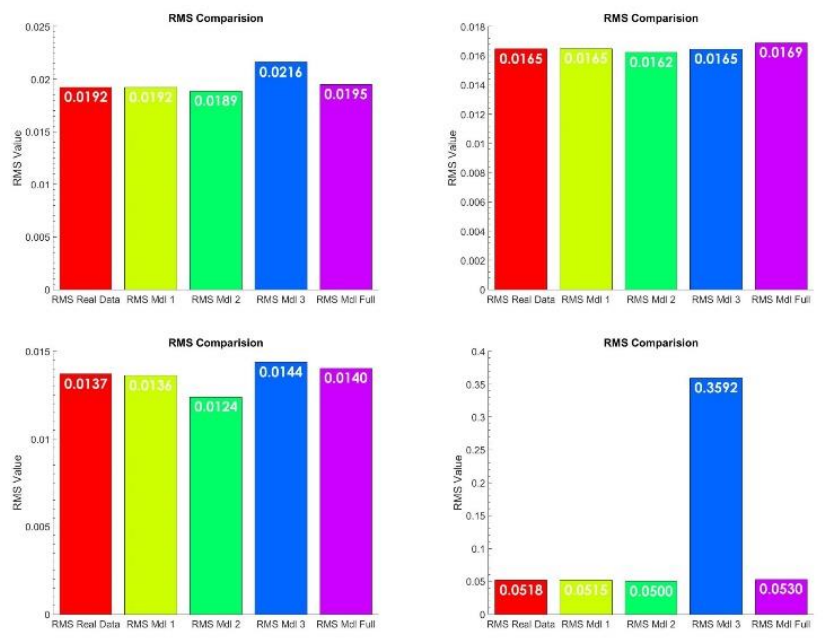

Figure 2: RMS error comparison for years 2011 (Top-Left), 2012 (Top-Right), 2013(Bottom-Left), 2014 (Bottom Right)

However, it can be assumed with some certainty that the electricity companies already use these factors in their models to provide the 
DAP prediction. Secondly, using higher order DAP models, only marginally improves on the RMS error, with second and third order showing the same.

Figure 2 show the results from the verification data from years 20112014. The data for 2014 was incomplete, so it should be disregarded. For the other years, the common trend is a small decrease of the RMS error with the first order and second order DAP, which means that the predictor is working as expected. However, starting with the third order models the error for the validation data set is increasing, which indicates overfitting to the training data. This also applies to the model with all factors included. Therefore, it is not recommended to rely on the third order or the full model for prediction.

It is clear when all the data results are analyzed, that it is possible to use DAP as an appropriate prediction for the RTP. Using linear regression, it may be possible to gain a very slight improvement using the first or second order predictor.

\section{Summary of the Stochastic Dynamic Programming Controller (SDPM)}

The control is based on two states in discrete-time. The state of charge (SoC) and the grid-cost (cost of electricity which changes over time due to market forces and demand) are these two states. The variable of concern, to the user apart from these states is time of charge i.e. needing a certain $\mathrm{SoC}$ in the battery by a certain time. The basic problem of 'when to charge?' assumes day-ahead prices are perfect, therefore we know the price in advance. It has one control variable the charging power $u$. The power is subject to two constraints: it cannot be negative and there is a constant maximum power $\bar{u}$ such that $u \in[0, \bar{u}]$. The battery state and total cost (output) both integrate (accumulate) over time and the only difference is the coefficient. It can be simply visualized as shown in figure 3 . The problem is explained in depth in a previous study [13].

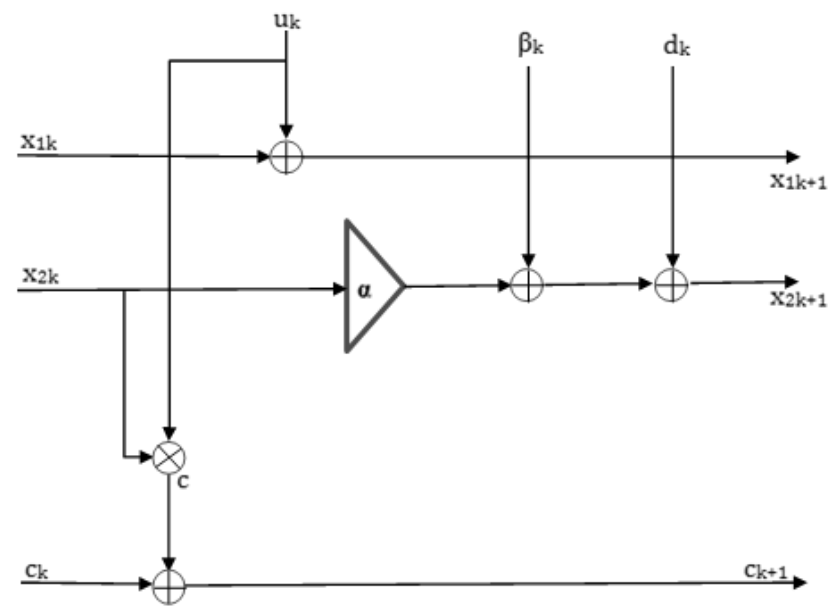

Figure 3: Diagrammatic Representation of the Basic Problem

In each time step $T s$ :

$\boldsymbol{x}_{\boldsymbol{I}}$ is the battery $\mathrm{SoC}$ (state)

$\boldsymbol{x}_{2}$ is the grid cost (state)

$c$ is the cost at each time step

$\boldsymbol{u}$ is the input (here: charge power)

$\boldsymbol{d}$ is the random disturbance causing price fluctuations
The controller tries to pick the cheapest hours to charge over the chosen charging period, thus providing a lowest average charging cost. However, this is based on the aforementioned assumption about the validity of the DAP prediction.

To describe this nature of the electricity grid we can call the price 'spikes' as events and associate a probability to the occurrence of these events. Describing the cost model as a standard first order linear process where:

$\boldsymbol{d}$ is an uncorrelated random variable with a normal distribution. It can be possible to define this as Gaussian and may make solutions more accurate but this needs to be tested after the simulation stage

$\boldsymbol{c}_{\boldsymbol{k}+\mathbf{1}}$ is the cost (calculation for time-step $k+1$ )

$\boldsymbol{\alpha}$ is the decay factor can be calculated by performing a correlation of the hourly prices

$\boldsymbol{\beta}$ is the disturbance factor can be calculated by the difference in the hourly prices

$\boldsymbol{P}_{\boldsymbol{e v}}$ is the probability of an event occurring

$\boldsymbol{\gamma}$ is the effect of an event

We get:

$$
c_{k+1}=\left\{\begin{array}{c}
\alpha c_{k}+\beta_{k} \cdot d \mid \text { no event }\left(d \geq P_{e v}\right) \\
\alpha c_{k}+\beta_{k} \cdot d+\gamma \mid \text { event }\left(d<P_{e v}\right)
\end{array}\right.
$$

The optimal solution, is defined as providing the lowest expected cost $E\langle J\rangle$ of deciding whether to charge or not, is difficult to find. A Dynamic Programming approach has been used to solve this. The approach is discussed in [12].

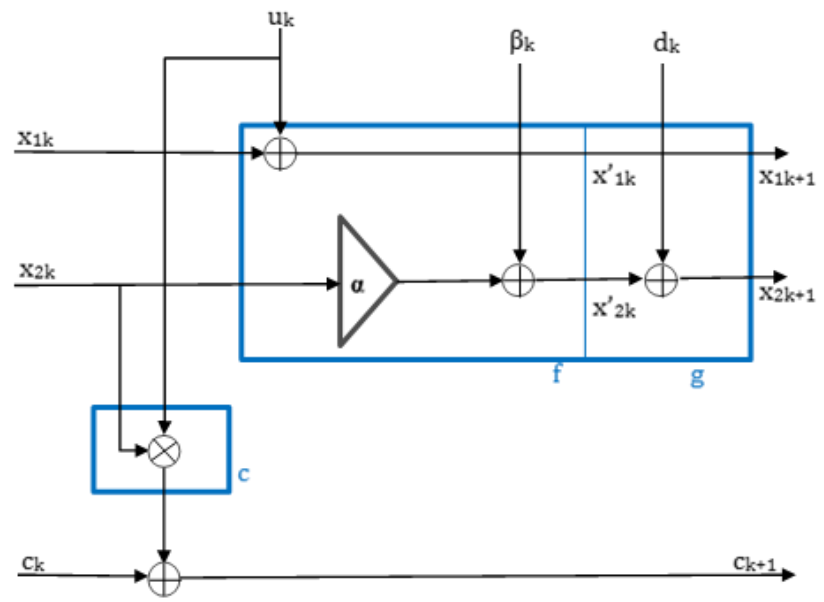

Figure 4: Stochastic Controller Approach with Dynamic Programming

The deterministic controller, provides a suboptimal solution. Once, the dynamic nature of the state variables are taken into consideration, the problem becomes a lot more complex. In this case, the varying nature of the grid-cost is important to the decision made by the controller and can be termed as the state which is affected by the stochastic disturbance. Therefore, this problem turns into a 2 state dynamic problem with a stochastic element. This is shown in figure 4.

Page 5 of 12

$10 / 19 / 2016$ 
A stochastic element (a random disturbance with a set probability of occurring), the disturbance is considered to account for unexpected price changes or spikes. This is computed prior to a dynamic programming iteration which attempts to achieve an optimal control strategy (optimization). This optimal control strategy is provided to a forward simulation to calculate the final cost of charging the vehicle. The SDPM algorithm with the model functions $f$ and $c$ (fig. 4 ) is described below. The stochastic dynamic programming controller (SDPM) is explained in depth in a previous study [12].

Algorithm 3: Stochastic Dynamic Programming

- State: $\boldsymbol{x}$ (here $X_{1}-$ SoC and $X_{2}-$ grid-cost)

- Control: $\boldsymbol{u}$ (here Charge Power)

- $\quad$ Grid size for all states

- Model functions: $\mathrm{f}$ and c here:

$$
\begin{gathered}
x_{1, k}^{\prime}=x_{1, k}+\mathrm{u}_{\mathrm{k}} \\
\mathrm{x}_{2, \mathrm{k}}^{\prime}=\alpha x_{2, k}+\beta_{k} \\
c_{k}=x_{2, k}+R u^{2}
\end{gathered}
$$

(where $R$ are the resistive losses within the battery)

- Final state cost: $J_{N+1}^{*}$ for all states $\boldsymbol{x}_{k+1}$ on the grid (here: $J_{N+1}=c_{N+1}\left(x_{N+1}\right) \times k$, where $c_{N}$ is the distance from the admissible set, and $k$ is a penalty factor)

- The stochastic model g here:

$$
\begin{gathered}
x_{1, k+1}=x_{1, k}^{\prime} \\
\mathrm{x}_{2, \mathrm{k}+1}=x_{2, k}^{\prime}+d_{k}
\end{gathered}
$$

- Time horizon: $\mathrm{N}$

1. State and input grids are created from discretization limits

2. Iterate over the time horizon backwards $k=N \ldots 1$

2.1 The expected cost function using the stochastic function $\mathrm{g}$ is calculated

$$
J_{k}^{\prime}\left(x_{k}^{\prime}\right)=E\left\langle J_{k+1}^{*}\left(x_{k+1}\right)\right\rangle
$$

\subsection{For each state $x_{k}$}

\subsubsection{For each input $\boldsymbol{u}_{k}$}

2.1.1.1 Evaluate the Model:

$$
\begin{aligned}
& x^{\prime}{ }_{k}=f\left(x_{k}, u_{k}\right) \\
& c_{k}=c\left(x_{k}, u_{u}\right)
\end{aligned}
$$

\subsubsection{Interpolate the remaining cost}

$J_{k}^{\prime}\left(x_{k}^{\prime}\right)$ Based on $x_{k}^{\prime}$ and the cost at the state grid points.

\subsubsection{Calculate the total cost}

Based on the step cost and the remaining cost

$$
J_{k}\left(x_{k}, u_{k}\right)=c_{k}\left(x_{k}, u_{k}\right)+J_{k}^{\prime}\left(x_{k}^{\prime}\right)
$$

2.1.2 Find the best input $u_{k}^{*}\left(x_{k}\right)$ that produces the lowest cost $J_{k}\left(x_{k}, u_{k}^{*}\right)=J_{k}^{*}\left(x_{k}\right)$

3. Optimal Charging Simulation

3.1 For the forward simulation, define the initial state $x_{1}$

3.2 Iterate over the time horizon forwards $k=1 \ldots N$

3.2.1 Evaluate the model

$$
x_{k+1}=f\left(x_{k}, u_{k}^{*}\left(x_{k}\right)\right)
$$

\section{Case Study using Illinois Smart Meter Example}

This study tries to validate the use of the SDPM by calculating the potential benefit to the vehicle user on a smart meter tariff in Illinois, by minimizing the cost of charging over a given period of time.

The case assumes the use of a typical electric vehicle, the base model Nissan LEAF, being used for typical work commute during the week and in-city driving during the weekend (for example, to visit the departmental store). The car is driven to work at $7 \mathrm{am}$ and back home at $5 \mathrm{pm}$ with an assumed total mileage of 50 miles, with each journey taking one hour. Whilst the stated NEDC mileage of the vehicle is up to 84 miles, a maximum of 70 possible miles are considered for a real world situation, whilst using air-conditioning and other amenities in the vehicle. The user plugs in the vehicle for charging at home at $5 \mathrm{pm}$, and charging is possible till $7 \mathrm{am}$ next morning when the vehicle is needed for commute to work. The electricity is provided by ComEd, with two tariffs considered, the day-ahead tariff, where prices are set at $5 \mathrm{pm}$ for the following day and real-time pricing available the hour before, on the day. Retrospectively, the history of these prices is available. We have five years of price data but for the purposes of discussing the results, one year is selected. The charging station, would be a level $2 \mathrm{AC}$ charging station with a typical output between $3.6 \mathrm{~kW}$ and $7.2 \mathrm{~kW}$, as defined in SAE $J 1772$ (2012 revision) standards[23]. Table 4, shows all the parameters used for the case.

Table 4: Baseline Parameters for Case Study

\begin{tabular}{|l|l|l|}
\hline \multicolumn{1}{|c|}{ Constant } & \multicolumn{1}{c|}{ Value } & \multicolumn{1}{c|}{ Unit } \\
\hline Vehicle & Nissan LEAF & - \\
\hline Battery Size & 24 & $\mathrm{kWH}$ \\
\hline Max-Charging Power & 6 & $\mathrm{~kW}$ \\
\hline Mileage of each journey & 50 & $\mathrm{miles}$ \\
\hline Probability of even occurring & 5 & $\%$ \\
\hline Decay constant & 0.7 & - \\
\hline Resistive Losses & 0 & Ohms \\
\hline Lowest allowed State of Charge & 20 & $\%$ \\
\hline
\end{tabular}

\section{Proving SDPM over a Single Day}

The SDPM is given a horizon of 31 hours, over which it must react to the charging profile of the user. This reflects the availability cycle of the price data, starting at 17:00 hours and also helps to see the reaction of the strategy to the requirements of the user profile discussed above. It must allow a maximum charge of the vehicle by the end of the horizon, by minimizing the average cost of charging but never let the battery fall below $20 \%$ (a cost penalty of 10 cents to the final cost is added in case of violation as a test). The baseline

Page 6 of 12 
assumes, we are able to give the DAP for optimization and RTP for the simulation within the SDPM.
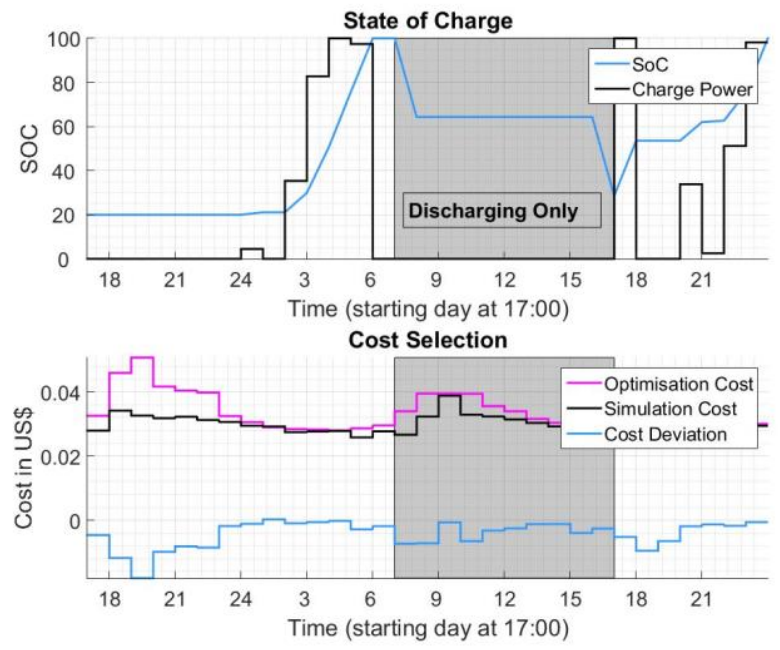

Figure 5: Charging with SDPM over a single day

Figure 5 shows the reaction of SDPM to the baseline laid out above. The white areas show the time when charging is allowed. The vehicle is then used at 07:00 hours and brought back and plugged in at 17:00 on the next day. No daytime charging is assumed in this study, and a review of user charging profiles justifying using this case is covered in [12], [24]. The use of the strategy successfully avoids the high prices right up to the moment they drop enough but also considering how much time is required to give sufficient charge to the vehicle. The total cost of charging is $\$ 1.0105$.
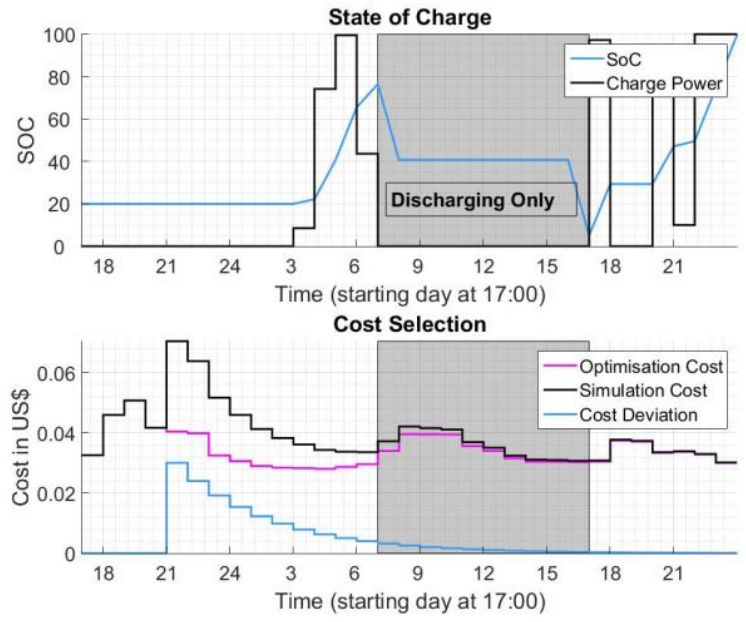

Figure 6: Charging with SDPM, reaction to an unexpected positive price event

Figure 6 shows the reaction of the strategy to an unexpected event in the fifth hour of the horizon. The price jumps by 3 cents, causing a parabolic decay in price over the coming hours. The strategy therefore waits as much as it can to avoid the increased prices, until the moment it has to allow charging, for sufficient SoC before the vehicle is driven for its next journey. During the second charging

Page 7 of 12 period it successfully completes a full charge of the battery. The total cost is $\$ 1.1819$.
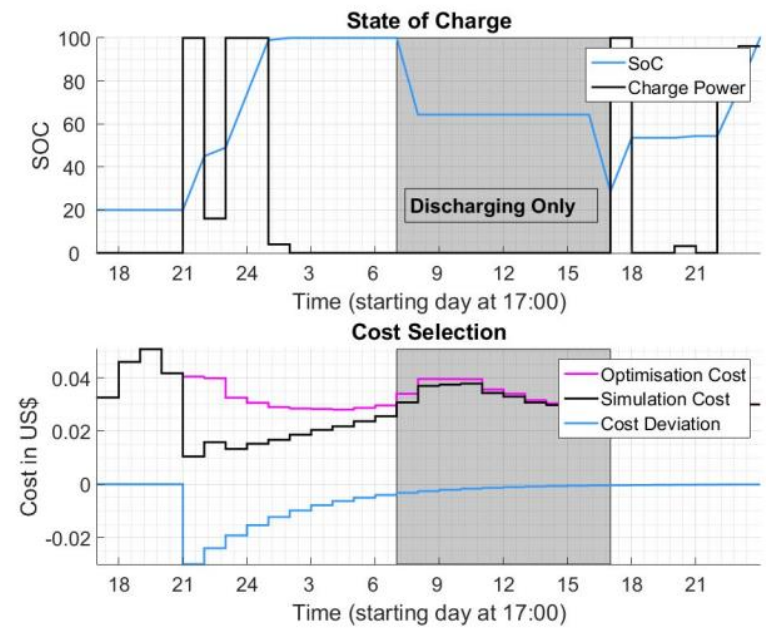

Figure 7: Charging with SDPM, reaction to an unexpected negative price event

Figure 7 shows the reaction of the SDPM to a similar but negative event, making the price fall by 3 cents. The strategy reacts instantly and charges the vehicle fully during this period, clearly saving considerably on the total cost of charging over the night. The total cost is $\$ 0.7820$.

Alongside the situation of a price-event or spike, it is interesting to consider a constant deviation situation. If both, the optimization and simulation costs are the same, the deviation would be zero (say in the case of both prices being given as DAP) but the reaction to a constant positive and negative deviation would help prove the validity of the strategy in a condition where it might have to take a penalty no matter what.
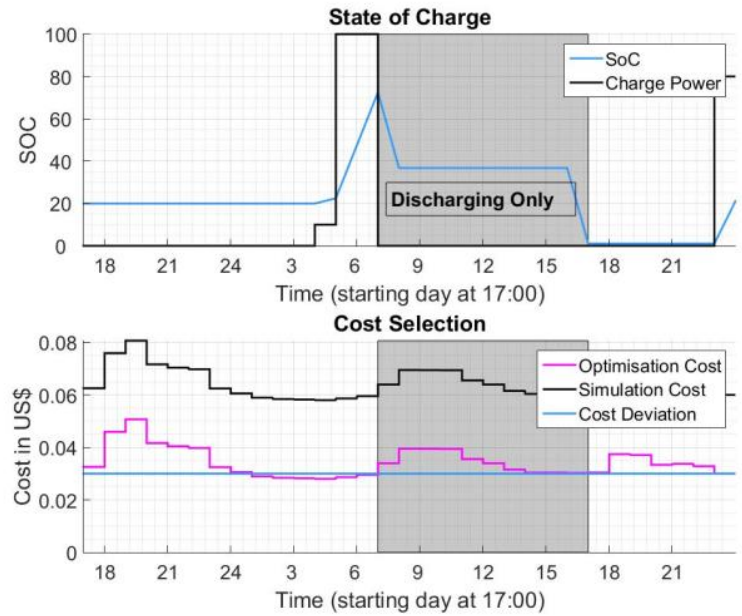

Figure 8: SDPM reaction to positive constant cost deviation

Figure 8 shows a hypothetical situation (on the same day) with constant positive cost deviation of 3 cents. It reacts similarly to the positive event but in this situation over the entire horizon of available prices it has to react the same way. Taking the first charging period, 
which would be of interest when needing charge at 07:00; it charges the vehicle enough by 'panicking' at the end, which ensures there is just enough SoC for the next day's journeys and the battery is just empty at 17:00. It has to take the final cost penalty associated with allowing the battery to discharge below $20 \%$. It pushes up the cost of charging significantly to $\$ 1.0325$. If a higher cost penalty is given, the strategy would avoid letting the SoC fall below the threshold. The question of how high the penalty should be, is not easily answerable and tied in with the question, how much a lithium-ion battery should be discharged but the fact that the strategy doesn't allow it to ever fall below $0 \%$ is obvious yet important in this case. In any case, such a high constant deviation wouldn't occur in reality.
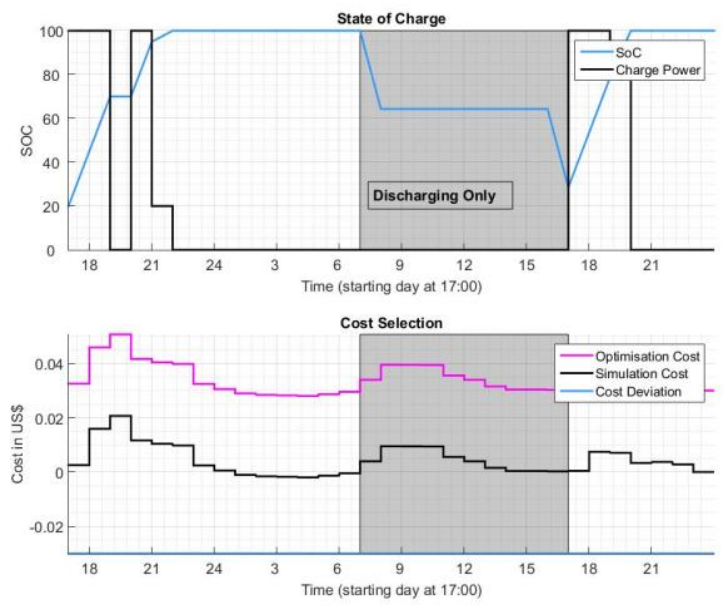

Figure 9: Reaction of SDPM to negative constant cost deviation

Figure 9 shows the reaction to a negative constant deviation and this observation is obvious. The SDPM charges at the first available opportunity and the total cost of charging is $\$ 0.2776$. However, it is worth noting that it does stop charging at 20:00, when the cost is actually a bit higher on average. It therefore, tries to minimize the cost even with a 3 cent advantage.

\section{Quantifying the use of the strategy: Advantage over a Year}

When running the strategy reaction over the year, the baseline parameters have been kept constant but a few price combinations have been considered to try and understand the reaction of the strategy, with a view to saving charging cost over the year. For comparison purposes, a fixed early charging strategy is used i.e. not using the SDPM, meaning the vehicle is charged when it is plugged in. After which, the following price combinations are used:

\section{Table 5: Price Combinations used by the SDPM}

\begin{tabular}{|c|c|}
\hline Price used for Optimization & Price used for Simulation \\
\hline Day Ahead Price & Day Ahead Price \\
\hline Day Ahead Price & Real Time Price \\
\hline $\begin{array}{c}\text { Linear Regression Predictor } \\
\text { output : DAP, DAP } 2 \text {, DAP }\end{array}$ & Real Time Price \\
\hline Real Time Price & Real Time Price \\
\hline
\end{tabular}

Using DAP for both, the deviation price is zero, and it is a simple case of the strategy reacting to the lowest price predictions but still considering the probability of an event spike. The DAP and RTP combination is as shown in the proof section, and is the possible price combination to provide the strategy. Then, the linear regression predictors are used for optimization to try and achieve a 'better DAP prediction' than the one provided whilst still using RTP for simulation. Finally, a non-causal combination is used with RTP being provided to the optimization algorithm. Of course, the last case is the 'ideal situation' where we have full knowledge of the future, which is never possible but it gives a good idea of how well the strategy can perform to achieve the goal.

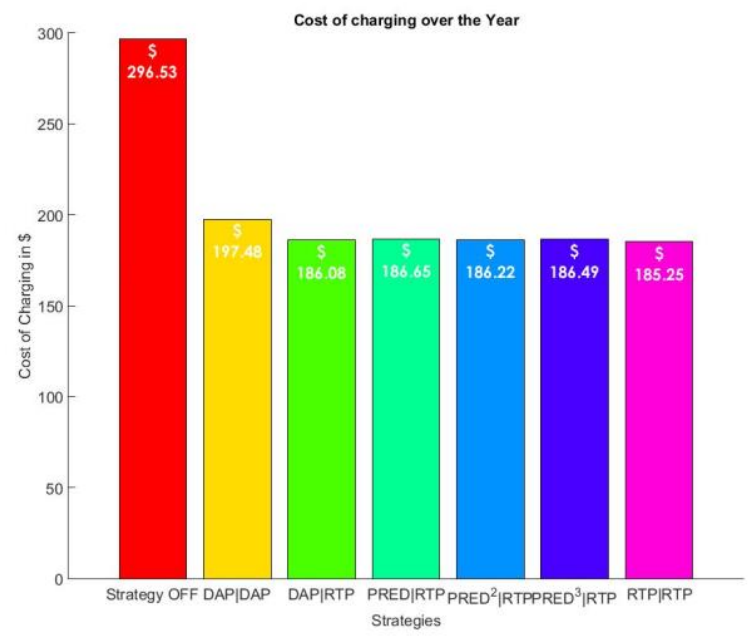

Figure 10: Cost savings over year 2011 cost data, when using SDPM with different price combinations

Figure 10 shows the final cost of charging over the year based on the 2011 year, electricity cost data. There is saving of $\$ \mathbf{9 9 . 0 5}$ when using the DAP-DAP combination and a larger saving of $\$ \mathbf{1 1 0 . 4 5}$ when using the DAP-RTP combination. This is as expected with a constant positive probability of a price spike. With a better indication of price, when using RTP for simulation, the controller is able to provide a lower cost.

The first order DAP predictor and RTP combination, actually gives a slightly higher final cost. The prediction is definitely not any better, but it isn't much worse either. With a different year and some difference in the parameters, like change in probability of a stochastic event, this could be higher or lower. It only confirms the results from the linear regression, in that there is a linear fit which diverges at higher prices and unexpected events. The second order DAP predictor gives a better result. However, the cost of charging over the year is still slightly higher than using just DAP. To try and understand this better, figure 11 shows the run over year 2013. 


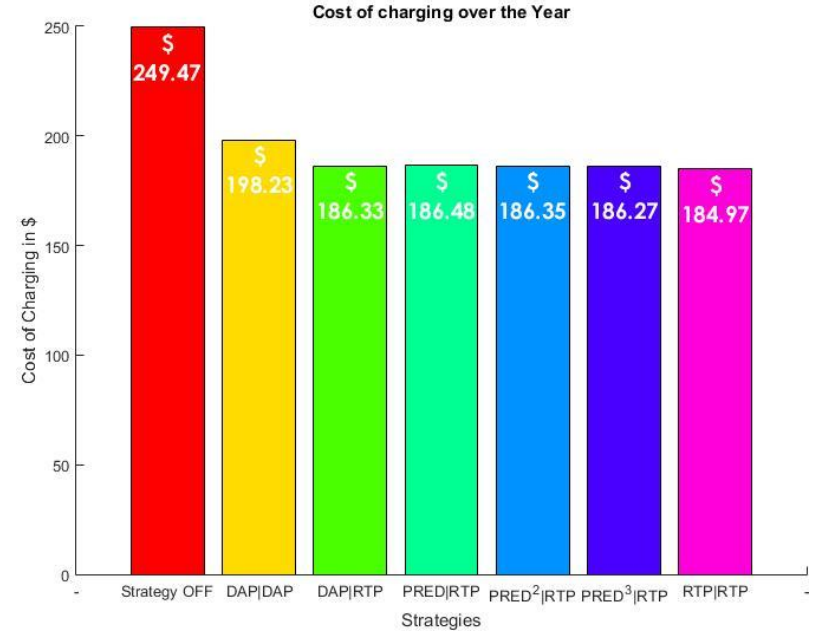

Figure 11: Cost savings over year 2013 cost data, when using SDPM with different price combinations

Whilst using the third order DAP for optimization gives a better prediction and hence lower cost in 2013, it doesn't in 2011. It is fair to conclude that the observations from the linear regression hold regarding the second order model being better in general but not reliable to be used over the DAP provided by the electricity company itself. It is worth noting that this may be true simply for ComEd's predictions, and the linear regression predictor, if performed on Amaren's data (with a lower correlation of 0.43 ) might have provided something different. In any case, the important question to answer is 'how much better?'

The last case of using RTP for optimization is ideal and unsurprisingly, it gives the lowest charging cost over the year when using the SDPM strategy. With full knowledge of the future, the strategy is able to react exactly to price changes, and no real speculative probability value needs to be given.

\section{Slow Charging Situation}

Fast charging is becoming the norm as EVs become more mainstream but SAE standards J1772, also mention a level 1 AC charging station with a typical output of $1.4 \mathrm{~kW}$. This is closer to a normal output from a home plug, using $120 \mathrm{~V}$ and it is interesting to consider whether a strategy like SDPM could save much over the year.
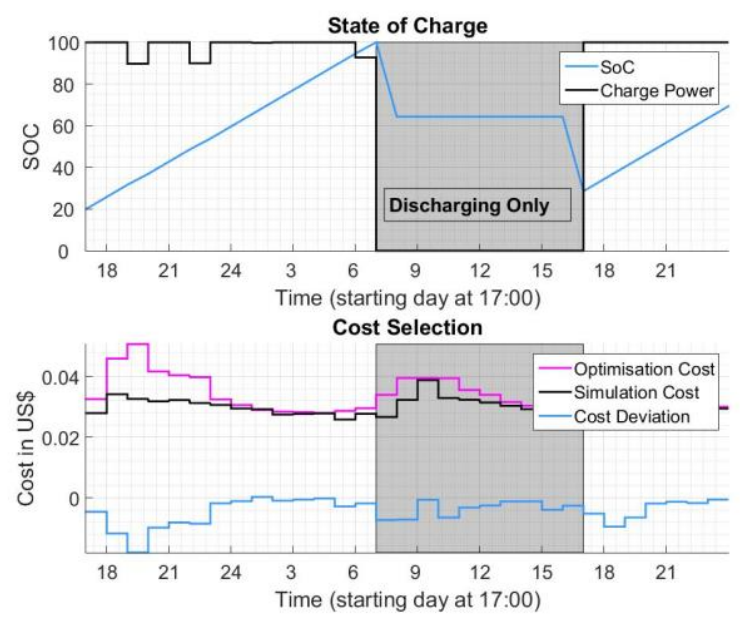

Figure 12: SDPM reaction using Slow Charging

Figure 12, shows that the strategy has to throttle the charging power where possible. It does so at particularly high prices on average, but it still has to charge almost all the time to allow for a required charge at the end of the period. However, the final cost of charging is $\$ 0.8613$ which is lower than the baseline fast charging cost for the same day of $\$ 1.0105$. This is clearly because less power is used although for a longer time owing to a lower overall cost but the charging takes significantly longer.

Looking at the overall cost of the year, this is reflected. Moreover, using the SDPM still achieves a lower cost compared to not using it. Using DAP prediction for both algorithms, $\$ 54.05$ is saved over the year, whereas the DAP and RTP combination achieves $\$ \mathbf{6 4 . 6 4}$

Although, the total cost is lower it is worth considering that neither in the baseline or slow charging case, resistive losses within the battery have been considered.

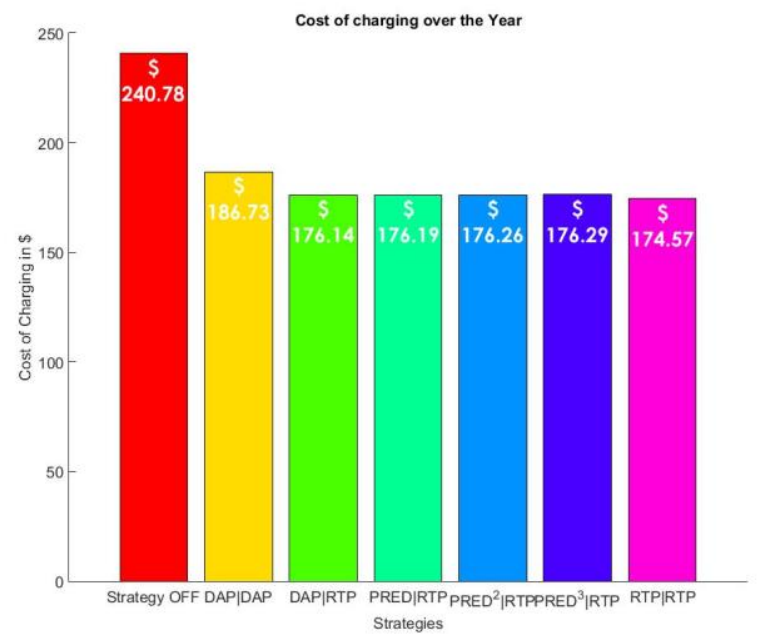

Figure 13: Year Cost Comparison for 2013 when using Slow Charge 


\section{Summary and Outlook}

The results show that the SDPM performs well with real price data from ComEd. It reacts intelligently to any prices spikes, positive or negative and minimizes the total charging cost when the probability of such events occurring is positive. When the predicted price agrees with the RTP tariff, it charges just a little before the lowest possible cost, which is reasonable to minimize the risk. In the constant deviation test, when the deviation is positive i.e. the price is constantly high, it is driven by the optimization constraints, and charges even at high cost to provide the required charge just-in-time. In any case of negative prices, it uses the opportunity to provide as much charge power as possible. Using the price data of 2011, using SDPM in the baseline price selection over the year saves $\$ \mathbf{1 1 0 . 4 5}$, or nearly half the cost, compared to a conventional charger.

The simple linear regression predictors, based on known factors from day ahead price data, show that DAP is already a good estimation of RTP. Some of the predictors show slight improvements in prediction, but there is also evidence of overfitting for the more complex predictors, resulting in worse performance on the verification data. The improvements in prediction cannot be demonstrated consistently on the charging simulation: there are slight cost saving in some years, but not in others. The conclusion is that for the considered data set, a regression prediction offers no significant advantage over the use of DA prices to predict RTP for the purpose of scheduling grid friendly charging.

A strategy like this can be very useful in future with EVs, for the vehicle owners. This would automate the procedure of charging at the right moments to take advantage of times of low electricity usage and in turn cheaper prices. However, it would be a matter of a real world study to see the effect this would have on the grid prices itself if many EVs were to use this strategy in tandem, potentially increasing the load on the grid simultaneously. The fairly low computational complexity of this approach presents an opportunity to load it on embedded systems and be present on either side: the vehicle charging electronics or within the charging station itself. Therefore, it is potentially useful to more than just one stakeholder i.e. the vehicle owner or it could be of advantage to charging stations or charging station manufacturers. The next logical step would be to perform such an experiment, embedding the control strategy on power electronics and testing its reaction to grid price changes. It would have to be performed along with an electricity provider who is allows the reading and transmission of their historical and current predicted price data to the system.

\section{References}

[1] A. Zubaryeva, C. Thiel, E. Barbone, and A. Mercier, "Assessing factors for the identification of potential lead markets for electrified vehicles in Europe: expert opinion elicitation," Technol. Forecast. Soc. Change, pp. 1-16, Jul. 2012.

[2] “World energy outlook 2009," IEA (International Energy Agency), Paris, 2009.

[3] "Nissan LEAF website." [Online]. Available: https://www.nissan.co.uk/vehicles/newvehicles/leaf/charging-range.html.

[4] J. E. Kang and W. W. Recker, "An activity-based

Page 10 of 12 assessment of the potential impacts of plug-in hybrid electric vehicles on energy and emissions using 1-day travel data," Transp. Res. Part D Transp. Environ., vol. 14, no. 8, pp. 541-556, Dec. 2009.

[5] “Tesla Motors Website." [Online]. Available: https://www.tesla.com/en_GB/models.

[6] P. Bauer, Y. Z. Y. Zhou, J. Doppler, and N. Stembridge, "Charging of electric vehicles and impact on the grid," MECHATRONIKA, 2010 13th Int. Symp., 2010.

[7] K. Clement-Nyns, E. Haesen, and J. Driesen, "Analysis of the impact of plug-in hybrid electric vehicles on residential distribution grids by using quadratic and dynamic programming," World Electr. Veh. J., vol. 3, 2009.

[8] J. Meyer, S. Hähle, P. Schegner, and C. Wald, "Impact of electrical car charging on unbalance in public low voltage grids," in Proceeding of the International Conference on Electrical Power Quality and Utilisation, EPQU, 2011, pp. 635-640.

[9] P. Stroehle, S. Becher, S. Lamparter, A. Schuller, and C. Weinhardt, "The impact of charging strategies for electric vehicles on power distribution networks," in 2011 8th International Conference on the European Energy Market, EEM 11, 2011, pp. 51-56.

[10] L. Zhang, T. Brown, and G. S. Samuelsen, "Fuel reduction and electricity consumption impact of different charging scenarios for plug-in hybrid electric vehicles," J. Power Sources, vol. 196, pp. 6559-6566, 2011.

[11] Z. Duan, B. Gutierrez, and L. Wang, "Forecasting plug-in electric vehicle sales and the diurnal recharging load curve," IEEE Trans. Smart Grid, vol. 5, no. 1, pp. 527-535, 2014.

[12] S. Mody and T. Steffen, "Optimal Charging of Electric Vehicles using a Stochastic Dynamic Programming Model and Price Prediction," SAE Int. J. Passeng. Cars - Electron. Electr. Syst., no. 8(2), pp. 379-393, 2015.

[13] S. Mody and T. Steffen, "Optimal Charging of EVs in a Real Time Pricing Electricity Market," SAE Int. J. ..., 2013.

[14] M. Shahidehpour, H. Yamin, and Z. Li, Market Operations in Electric Power Systems. 2002.

[15] X. S. Han, L. Han, H. B. Gooi, and Z. Y. Pan, "Ultra-shortterm multi-node load forecasting - a composite approach," IET Gener. Transm. Distrib., vol. 6, no. 5, p. 436, May 2012.

[16] T. Hong, J. Wilson, and J. Xie, "Long term probabilistic load forecasting and normalization with hourly information," IEEE Trans. Smart Grid, vol. 5, no. 1, pp. 456-462, 2014.

[17] D. Singhal and K. S. Swarup, "Electricity price forecasting using artificial neural networks," Int. J. Electr. Power Energy Syst., vol. 33, no. 3, pp. 550-555, 2011. 
[18] L. Wu and M. Shahidehpour, "A hybrid model for integrated day-ahead electricity price and load forecasting in smart grid," IET Gener. Transm. Distrib., vol. 8, no. 12, pp. 19371950, 2014.

[19] H. Allcott, "Rethinking real-time electricity pricing," Resour. Energy Econ., vol. 33, no. 4, pp. 820-842, Nov. 2011.

[20] The Brattle Group, Freeman Sullivan \& Co, and Global Energy Partners, "A National Assessment of Demand Response Potential," Ferc, p. 254, 2009.

[21] R. R. Pricing, "Bringing Residential Real-Time Pricing to Scale in Illinois : Policy Recommendations," pp. 2-5, 2009.

[22] “ComEd Electricity Website." [Online]. Available: https://www.comed.com/Pages/default.aspx.

[23] Hydro Quebec, "Electric Vehicle Charging Stations Technical Installations Guide," 2012.

[24] S. Mody and T. Steffen, "Optimal Charging of EVs in a Real Time Pricing Electricity Market," SAE World Congr. 2013, 2013.

\section{Contact Information}

Sagar Mody: S.Mody@lboro.ac.uk

Thomas Steffen: T.Steffen@lboro.ac.uk

\section{Definitions/Abbreviations}

BEV battery electric vehicle

DA day-ahead pricing

HEV hybrid electric vehicle

ICE internal combustion engine

MPC model (based) predictive control

RT/RTP real time pricing

PHEV plugin-in hybrid electric vehicle

SOC (battery) state of charge

SD standard deviation

V2G vehicle to grid

EV Electric vehicle

DP Dynamic programming

SDP Stochastic dynamic programming

ToC Time of charge (required time)

SoC State of charge (required) 
Page 12 of 12

10/19/2016 